Trinity University

Digital Commons @ Trinity

Engineering Faculty Research

Engineering Science Department

$9-2013$

\title{
Correlation for the Convective and Diffusive Evaporation of a Sessile Drop
}

Peter Kelly-Zion

TrinityUniversity, plkelly@trinity.edu

J. Batra

Trinity University

Christopher J. Pursell

Trinity University, cpursell@trinity.edu

Follow this and additional works at: https://digitalcommons.trinity.edu/engine_faculty

Part of the Engineering Commons

\section{Repository Citation}

P.L. Kelly-Zion, J. Batra, C.J. Pursell, "Correlation for the convective and diffusive evaporation of a sessile drop," International Journal of Heat and Mass Transfer, 64(2013) pp. 278-285. http://dx.doi.org/10.1016/j.ijheatmasstransfer.2013.04.051

This Post-Print is brought to you for free and open access by the Engineering Science Department at Digital Commons @ Trinity. It has been accepted for inclusion in Engineering Faculty Research by an authorized administrator of Digital Commons @ Trinity. For more information, please contact jcostanz@trinity.edu. 


\title{
Correlation for the Convective and Diffusive Evaporation of a Sessile Drop
}

\author{
P.L. Kelly-Zion ${ }^{\mathrm{a}, *}$, J. Batra ${ }^{\mathrm{a}}$, and C.J. Pursell ${ }^{\mathrm{b}}$ \\ ${ }^{a}$ Department of Engineering Science \\ Trinity University, San Antonio 78212, United States \\ ${ }^{\mathrm{b}}$ Department of Chemistry \\ Trinity University, San Antonio 78212, United States
}

\begin{abstract}
A simple correlation is developed to compute the evaporation rates of sessile drops and small puddles which are evaporating under the influences of both diffusion and natural convection of the vapor-air mixture surrounding the drop. The correlation is based on experiments conducted with eight hydrocarbons, which provide a factor of 16.6 variation in volatility as indicated by the equilibrium vapor pressures, a factor of 3.6 variation in molecular mass, and a factor of 2.2 variation in mass diffusivity, and thus the correlation is applicable for liquids having a broad range of properties. The correlation predicts the evaporation rates to within a root-mean-square (RMS) error of $6.5 \%$ over the broad range of conditions. Limitations of the correlation are investigated, and when one of the species is excluded, the RMS error is reduced to $4.9 \%$.

There are two main differences between this new correlation and the correlations that have been published previously. The first difference is the new correlation reduces to an expression for diffusion-limited evaporation as the density difference between the vapor-air mixture at the surface of the drop and the ambient air becomes negligible, or when the drop size becomes very small. The second difference is the form of the dependency on the density difference ratio, which in previous correlations is obtained solely through the Rayleigh number $(R a)$. This new correlation contains a term which represents the influence of natural convection on the evaporation rate and this term provides insight into the nature of the coupling of the diffusive and convective transport of the vapor.
\end{abstract}

\section{Keywords}

Evaporation; natural convection; diffusion; sessile drop

\section{Nomenclature}

a correlation constant

c correlation constant

$D \quad$ diffusion coefficient

E mass evaporation rate

$E_{D} \quad$ mass evaporation rate for diffusion-limited evaporation

$g \quad$ gravitational acceleration

Gr Grashof number

$h_{f g} \quad$ enthalpy of vaporization

$h_{m} \quad$ mass transfer coefficient

$M \quad$ molecular weight of the evaporating species

$\dot{m}_{e} \quad$ evaporative flux

$n \quad$ correlation exponent

$P \quad$ ambient pressure

$P r \quad$ Prandtl number

$P_{v} \quad$ equilibrium vapor pressure

$R \quad$ drop radius

$R_{u} \quad$ universal gas constant

Sc Schmidt number

Sh Sherwood number

$S h_{D} \quad$ Sherwood number for diffusion-limited evaporation

$T \quad$ ambient temperature

$R a \quad$ Rayleigh number

$z \quad$ counter-diffusion correction factor

Greek Symbols

$\alpha \quad$ thermal diffusivity

* Corresponding author: Tel.: 1210999 7518; fax: 12109998037

E-mail address: peter.kelly-zion@trinity.edu (P.L. Kelly-Zion) 


$\begin{array}{ll}\rho & \text { mass density } \\ \mu & \text { dynamic viscosity } \\ v & \text { kinematic viscosity } \\ \phi & \text { relative humidity }\end{array}$

$\begin{array}{ll}\text { Subscripts } \\ a & \text { air } \\ c & \text { computed } \\ m & \text { mixture or measured } \\ s & \text { located at the surface of the drop } \\ \infty & \text { located far from the drop } \\ g & \text { saturated air }\end{array}$

\section{Introduction}

Sessile drop evaporation has an important role in many technical applications, including spray cooling, painting and coating, and some lab-on-a-chip designs. Sophisticated computational models have been developed to analyse and predict evaporation processes, and these models have improved greatly the understanding of the important physical phenomena. However, because of their complexity the models are difficult to implement. For example, for the relatively simple case of diffusion-limited evaporation, researchers use computational techniques to solve the pertinent conservation equations for the evaporation rate [14]. Alternatively, an analytical expression for diffusion-limited evaporation can be obtained through the use of the electrostatic analogy of a flat disc kept at constant potential [5-7]. To facilitate the inclusion of evaporation in the analyses of practical applications, it is desirable to have a correlation that is simple to implement and that is valid over a broad range of conditions, including those in which natural convection of the vapor is significant.

Few simple correlations have been published for evaporation in a still environment, for which the combined effects of vapor phase natural convection and diffusion control the evaporation rate (as opposed to forced convection for which there are a comparatively large number of published studies). This set of correlations is dominated by those developed specifically for water evaporation [8-15]. For the evaporation of liquids other than water, the heat and mass transfer analogy may be used but, to our knowledge, no simple, non-computational models have been developed for evaporation of sessile drops under conditions for which natural convection may be significant.

One of the methods used for correlating water evaporation data is to determine a relationship for the dimensionless evaporation rate represented by the Sherwood number $(S h)$ as a function of the Rayleigh number $(R a)$ and Schmidt number $(S c)$ in the form given by equation (1).

$$
S h=c \cdot S c^{1 / 3} \cdot R a^{n}
$$

The prefactor $c$ and the exponent $n$ are fitting constants. Bower and Saylor published an excellent review of correlations of this form and evaluated the ability of the correlations to fit their measurements, which were conducted with heated water in square tanks of widths between 15.2 and $60.9 \mathrm{~cm} \mathrm{[8,9].} \mathrm{After} \mathrm{accounting} \mathrm{for} \mathrm{slight} \mathrm{differences} \mathrm{in} \mathrm{the} \mathrm{definitions} \mathrm{of} S h$ and $R a$ and recomputing the correlations using uniform definitions, Bower and Saylor found significant differences in the prefactors, $c$, and the exponents, $n$, amongst four correlations for water evaporation under conditions of natural convection. Three of the four correlations were developed for the case in which the water vapor is less dense than the surrounding air, which results in a buoyancy-induced upward flow of the vapor $[8,9,11,12]$. One correlation was developed for the case in which the water vapor was colder and denser than the surrounding air, resulting in a buoyancy-induced downward flow of the vapor [10]. Despite the difference in the nature of the convective flow, the prefactor and exponent for this correlation are within the range of values of the correlations for the upward directed convective flow.

Jodat et al. measured the evaporation rate of heated water in a large pan for a range of conditions which include vapor transport by natural convection [14]. They correlated their results using different forms of Dalton's equation, given in general form by equation (2), and were able to obtain a good fit for $c=0.0069, n=1.105$, and $n^{\prime}=0.153$.

$$
\dot{m}_{e}=\frac{c\left(P_{v, s}-\varphi P_{v, \infty}\right)^{n}\left(\rho_{g, s}-\rho_{g, \infty}\right)^{n^{\prime}}}{h_{f g}}
$$

In equation (2), $\dot{m}_{e}$ is the evaporative flux in units of $\left[\mathrm{kg} / \mathrm{m}^{2} \mathrm{hr}\right], P_{v}$ is the saturated vapor pressure of water in [Pa] with the subscripts $s$ and $\infty$ indicating locations at the surface of the liquid and far from the surface, $\varphi$ is the relative humidity, $\rho_{\mathrm{g}}$ is the density of the saturated air in $\left[\mathrm{kg} / \mathrm{m}^{3}\right]$, and $h_{f g}$ is the enthalpy of vaporization in $[\mathrm{J} / \mathrm{kg}]$.

With the goal of developing a general correlation for the evaporation rate of a sessile drop that is applicable for a broad range of volatility, vapor density, and drop size, equations (1) and (2) both were applied to evaporation rate measurements of eight different hydrocarbons, given in Table 1 . The volatilities of these eight species, as measured by their equilibrium vapor pressures at $23^{\circ} \mathrm{C}$, vary by a factor of 16.6. The difference between the densities of the vapor-air mixture at the drop surface, $\rho_{m}$, and the ambient air, $\rho_{a}$, i.e. $\left(\rho_{m}-\rho_{a}\right)$, which is what drives the natural convection, varies by a factor of 26.5 . Since the molecular weights 
of the species are all greater than that of air, the buoyancy-induced flow is directed down and radially away from the surface of the drop. The drop radius was varied from $1 \mathrm{~mm}$ to $22 \mathrm{~mm}$. As drop size increases, the drop flattens and for large sizes the drop may be more accurately described as a puddle. However, for consistency, the term "drop" is used for all sizes.

Strong evidence of natural convection occurring during the evaporation of these hydrocarbon species is provided by schlieren videos, which indicate that a pancake-shaped vapor cloud forms above the evaporating drop and this cloud flows radially away from the drop by natural convection [16]. The flow of vapor is assumed to be similar to the thermally induced natural convection along the top surface of a chilled disk. As the drop size increases, the influence of natural convection seems to become stronger the measured evaporation rate for a $22 \mathrm{~mm}$ radius drop of 3-methylpentane (3MP) is $475 \%$ greater than a value predicted by a simple diffusion-limited model [17].

Finding that neither equation (1) nor (2) provides a satisfactory correlation to the evaporation rate data of the hydrocarbon species, a new correlation was developed for the evaporation of pinned, sessile drops under the conditions of combined diffusive and naturally convective transport of the vapor. The new correlation has a form similar to that of equation (1) but which contains two additional terms. One of the additional terms provides a limiting result that is equivalent to diffusion-controlled evaporation, and the other additional term improves the dependency on the density difference. The dependencies of the new correlation provide insight into the coupled nature of diffusion and natural convection for the species studied.

\section{Methods}

\subsection{Experimental Methods}

The experimental method is described in detail in [17] and is summarized below.

Drops were injected onto the center of a flat, aluminium substrate of radius of $65 \mathrm{~mm}$. To control the size of the drop, a slightly raised, disk-shaped platform was located at the center of the substrate which provides a circular edge along which the drop attaches, as shown in Fig. 1. The drop remains attached during the evaporation process, and therefore the contact area remains constant, until the liquid volume becomes insufficient to cover the platform at which time the drop pulls from the edge. Only measurements taken during the time a drop remains fully attached are used in the analyses. The height of the platform is $1 / 2 \mathrm{~mm}$ for drop radii less than or equal to $4 \mathrm{~mm}$, and is $1 \mathrm{~mm}$ for larger radii.

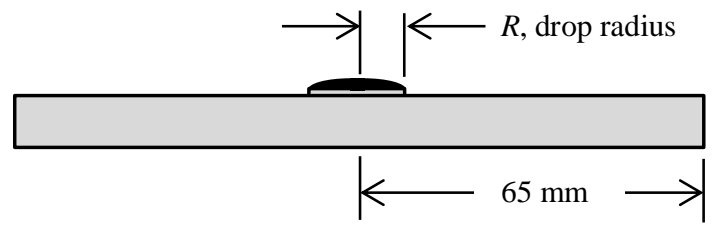

Figure 1. Schematic of a profile view of a sessile drop on the disk-shaped substrate. The drop is attached around the circular periphery of a short platform located at the center of the substrate, and in this way the drop size is maintained during evaporation.

Sparrow et al. found that the water evaporation rates from their experiments were dependent upon the ratio of the diameter of the flat frame surrounding their pan of water to the diameter of the pan when this ratio is less than three [10]. When the diameter ratio is equal to three or more, it no longer affects the evaporation rate, and presumably the measured evaporation rate is equivalent to what would be measured for water on an infinite horizontal surface. Therefore, it is important to note that the ratio of the substrate diameter to drop diameter for the experiments reported here is 2.95 and larger.

The drops were generated by injecting a controlled volume of liquid onto the substrate using a pipettor. The substrate and liquid were maintained at room temperature, which was $23.2 \pm 0.7^{\circ} \mathrm{C}$. The ambient pressure was $96 \pm 2 \mathrm{kPa}$.

To isolate the experiments from drafts in the room and to ensure an initially still environment, the substrate was contained in an enclosure of volume approximately $6200 \mathrm{~cm}^{3}$. Care was taken to vent the enclosure along the sides at the bottom in order to prevent the build-up of vapor while still isolating the experiment from drafts. Tests conducted with and without venting indicated that the evaporation rates of drops of radius $8 \mathrm{~mm}$ and larger reduced when the enclosure was not vented, whereas the evaporation rates of smaller drops were unaffected.

Two methods were used to measure the evaporation rate. For drops equal to or larger than $4 \mathrm{~mm}$ in radius, a simple gravimetric technique was used whereby the mass of the drop was measured over time by an analytical balance, which has a resolution of 0.1 $\mathrm{mg}$. The mass was measured at a rate of either 2 or $10 \mathrm{~Hz}$ and the evaporation rate was computed as the magnitude of the slope of the changing mass, making sure that only measurements acquired prior to the time when the drop pulls from the edge of the platform are used.

For drops having a radius of 1 or $2 \mathrm{~mm}$, the evaporation rates were slow enough that the resolution of the balance caused a stairstep pattern in the transient mass measurements. To avoid this problem, a shadowgraph imaging technique was used to measure the profile of the evaporating drop and the drop volume was computed from the profile. Images were recorded at a rate of 60 frames/sec and the evaporation rate was computed from the changing volume and the liquid density. To test the agreement of the 
gravimetric and imaging techniques, both were used to measure the evaporation rate of a hexane drop of radius 4 mm. The two results agree within 7\%, as discussed in [17]. The average percent variation in the shadowgraph results for a given species was $\pm 7 \%$ for $1 \mathrm{~mm}$ drops and $\pm 3 \%$ for $2 \mathrm{~mm}$ drops.

With only a few exceptions, the evaporation rates were constant during the evaporation process and thus showed no effect either of cooling of the drop surface or of a changing contact angle. However, for 1 and $2 \mathrm{~mm}$ drops, there was a gradual change in the evaporation rate, which typically resulted in a $10 \%$ variation from the mean during the experiment. In the worst case, there was a $20 \%$ variation. This variation is discussed in detail in reference [17] and is attributed to a large variation in the contact angle for the 1 and $2 \mathrm{~mm}$ drops during the evaporation process.

Each experimental condition was conducted at least three times, and generally five times, and the values reported are the averages of the multiple measurements.

Table 1 provides a comparison for the eight hydrocarbons used in this study of the equilibrium vapor pressures, gas diffusion coefficients in air, and differences in densities, relative to the air density, between the vapor-air mixture and the surrounding air, i.e. $\left(\rho_{m}-\rho_{a}\right) / \rho_{a}$. These property values are given for the average temperature and ambient pressure of the experiments, $23.3^{\circ} \mathrm{C}$ and $96 \mathrm{kPa}$. The equilibrium vapor pressures were computed using the Antoine equation, as given in Yaws [18], and the diffusion coefficients are computed according to a correlation by Fuller et al., which is given in [19]. The density of the vapor-air mixture is estimated using the ideal gas law and assuming the partial pressure of the vapor is equal to the equilibrium vapor pressure. While this assumption enables a simple technique to estimate the mixture density, in fact the vapor pressure is less than the equilibrium vapor pressure except very near the drop surface, and the computed mixture density represents an upper limit.

\begin{tabular}{|c|c|c|c|c|c|c|c|c|}
\hline Species & Acetone & 3MP & Hexane & Methanol & Cyclohexane & Isooctane & Heptane & Octane \\
\hline $\boldsymbol{P}_{\boldsymbol{v}}[\mathrm{kPa}]$ & 28.4 & 22.0 & 18.8 & 15.4 & 12.0 & 6.06 & 5.61 & 1.71 \\
\hline $\boldsymbol{D}\left[\mathbf{m m}^{2} / \mathbf{s}\right]$ & 11.1 & 8.00 & 8.00 & 16.7 & 8.16 & 6.86 & 7.36 & 6.86 \\
\hline$\left(\boldsymbol{\rho}_{\boldsymbol{m}}-\boldsymbol{\rho}_{\boldsymbol{a}}\right) / \boldsymbol{\rho}_{\boldsymbol{a}}$ & 0.30 & 0.45 & 0.39 & 0.017 & 0.24 & 0.19 & 0.14 & 0.052 \\
\hline
\end{tabular}

Table 1. Thermophysical properties of the hydrocarbon species at $23.3^{\circ} \mathrm{C}$ and $96 \mathrm{kPa}$.

\subsection{Correlation Development}

Prior to developing a new correlation, the results of previous correlations in the forms of Eqs. 1 and 2 were compared with our measurements. In both cases, the fitting constants were optimized to fit our measurements. For equation $2, \varphi$ is set to zero since no vapor was present far from the evaporating drop. The results are presented in Fig. 2 in terms of the percentage error between the computed evaporation rates, $E_{c}$, derived from Eqs. 1 and 2, and the measured evaporation rates, $E_{m}$. The plots show the errors for six of the species used in this study, which were measured over a range of drop sizes. The results for isooctane and octane are taken from a previous study, reference [16], which was conducted for a single drop size, $6.5 \mathrm{~mm}$ radius. The errors for isooctane and octane are given in Table 2. As shown in Fig. 2a, the error associated with Eq. 1 appears to be relatively insensitive to the drop size for radii larger than $4 \mathrm{~mm}$, but the error varies significantly from one species to another. This behavior suggests that the correlation could be improved by modifying its dependency on one or more of the physical properties of the species. The RMS error for Eq. 1 is $12.7 \%$.

\begin{tabular}{|c|c|c|}
\hline Species & $\begin{array}{c}\text { Error in Eq. 1 } \\
\left(\boldsymbol{E}_{\boldsymbol{c}}-\boldsymbol{E}_{\boldsymbol{m}}\right) / \boldsymbol{E}_{\boldsymbol{m}} \\
{[\boldsymbol{\%}]}\end{array}$ & $\begin{array}{c}\text { Error in Eq. 2 } \\
\left(\boldsymbol{E}_{\boldsymbol{c}}-\boldsymbol{E}_{\boldsymbol{m}}\right) / \boldsymbol{E}_{\boldsymbol{m}} \\
{[\boldsymbol{\%}]}\end{array}$ \\
\hline Isooctane & 21.2 & 53.4 \\
\hline Octane & 16.4 & 57.7 \\
\hline
\end{tabular}

Table 2. Percent error resulting from the use of Eqs. 1 and 2 to compute the evaporation rates of isooctane and octane for a drop radius of $6.5 \mathrm{~mm}$.

The results for Eq. 2, which are presented in Fig. 2b, indicate a substantially worse agreement with the measurements than what is given by Eq. 1. Not only does the error vary significantly from one species to another, the error also is drop size dependent. The RMS error for Eq. 2 is $70.1 \%$.

Due to the relative success of Eq. 1 (despite having one fewer fitting constant) and the fact that this equation contains terms that are important for both diffusion and natural convection, our approach was to modify the $S h$ - $R a$ relationship given by Eq. 1 in order to develop an improved correlation that is valid for a broad range of species and drop sizes. 


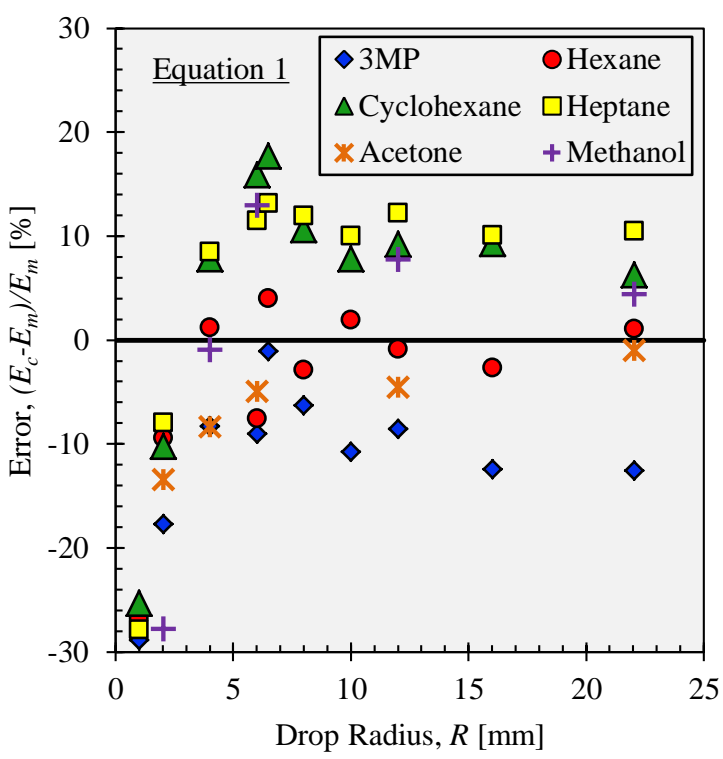

(2a)

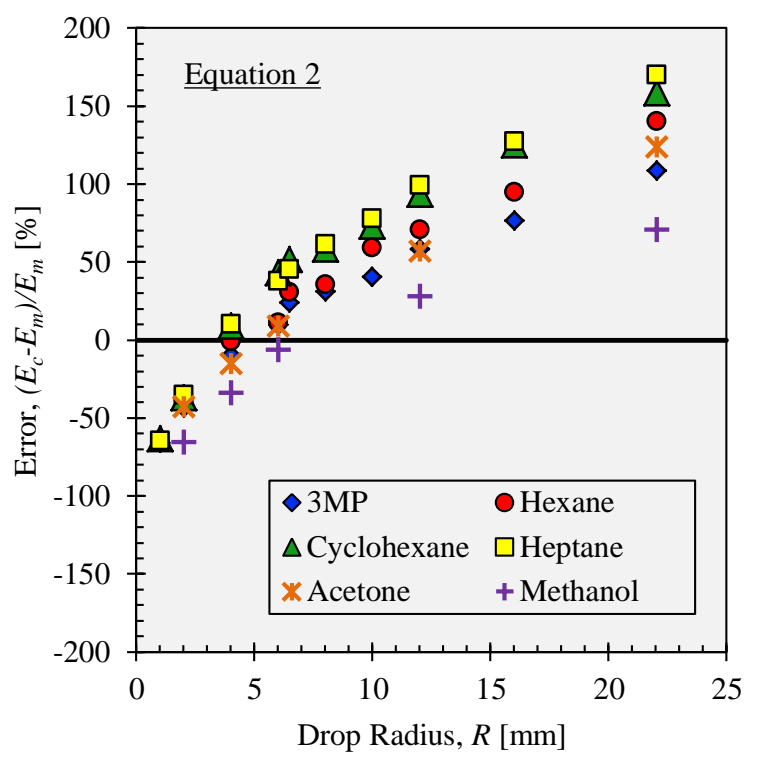

(2b)

Figure 2. Comparison of the percentage error in the computed evaporation rates from previously published correlations given by Eq. 1 (Fig. 2a) and Eq. 2 (Fig. 2b). The RMS errors are 12.7\% for Eq. 1 and 70.1\% for Eq. 2.

$S h$ and $R a$ are defined by Eqs. 3 and 4. To determine an expression for the mass transfer coefficient, $h_{m}$, the vapor concentration difference is taken to be equal to the saturated concentration at the drop surface since there is no vapor far from the drop. Furthermore, the saturated vapor concentration is computed using the ideal gas law and setting the partial pressure of the vapor equal to the equilibrium vapor pressure.

$$
\begin{aligned}
& S h=\frac{h_{m} R}{D}=\frac{E R_{u} T}{\pi R D P_{v} M} \\
& R a=\operatorname{GrPr}=\left[\left(\frac{\rho_{m}-\rho_{a}}{\rho_{a}}\right) \frac{g R^{3}}{v_{a}^{2}}\right]\left(\frac{v_{a}}{\alpha}\right)
\end{aligned}
$$

The definition of $R a$ given by Eq. 4 differs slightly from that used by Bower and Saylor [8, 9]. To simplify the expression, the ambient air density, $\rho_{a}$, is used in the denominator rather than an average of the saturated vapor and air densities as used by Bower and Saylor.

In trying to find a better correlation for our range of conditions, $R a$ was broken into component parts with each dimensionless part allowed to influence the correlation to a different degree through the use of independent exponents, as given by equation (5). In this way, the magnitude of the effect of the density difference ratio, which drives natural convection, is not constrained by the effect of drop size, i.e. the exponents $n_{1}$ and $n_{3}$ may be different, which is not the case when these two parameters are contained solely in $R a$ and thus are raised to the same exponent, as occurs in Eq. 1. Furthermore, the exponent on $S c\left(S c=v_{a} / D\right)$ through which the effect of diffusivity is introduced, is not constrained to the value $1 / 3$, which derives from the analogy to convective heat transfer. Since the experiments were conducted at the same temperature, $\operatorname{Pr}$ for air is constant $(0.708)$ and is embedded in the coefficient $c_{2}$. Another modification to the form of the $S h-R a$ relationship is the constant, $c_{l}$, which is added to enable the correlation to reduce to a nonzero value under conditions when there is no natural convection, i.e. when the evaporation rate is diffusion-limited.

$$
S h=c_{1}+c_{2}\left(\frac{\rho_{m}-\rho_{a}}{\rho_{a}}\right)^{n_{1}} S c^{n_{2}}\left(\frac{g R^{3}}{v_{a}^{2}}\right)^{n_{3}}
$$

The value of the constant $c_{l}$ was found to be 1.25. Considering a condition in which natural convection is not occurring, for example when $\rho_{m}=\rho_{a}$, then equation (5) gives $S h=c_{l}$. The definition for $S h$ (Eq. 3) may be used to express the evaporation rate, $E$, in terms of the constant $c_{1}$. One may also use Stefan's solution for diffusion from a flat circular disk to compute the diffusionlimited evaporation rate, $E_{D}$, which is given by Eqs. 6 and 7 [6]. z is the counter-diffusion correction factor. Using Eqs. 3 and 6 , the expression $S h=c_{1}$ may be transformed to the expression given by Eq. 8. The counter-diffusion factor, z, is approximately equal to 1 , varying between 1.01 (octane) and 1.14 (3MP) for the conditions of the experiments and so the fact that $c_{1}$ is 1.25 indicates that the correlation approaches the value for diffusion-limited evaporation for cases when natural convection is negligible, as would be expected. 


$$
\begin{aligned}
& E_{D}=\frac{4 R D M P_{v}}{R_{u} T} Z \\
& z=\frac{P}{P_{v}} \operatorname{Ln}\left[\frac{1}{\left(1-\frac{P_{v}}{P}\right)}\right] \\
& E=\frac{c_{1} \pi E_{D}}{4 z}=0.982 \frac{E_{D}}{z} \approx E_{D}
\end{aligned}
$$

To improve the correlation, the constant $c_{l}$ was replaced with the Sherwood number based on diffusion-limited evaporation, $S h_{D}$, which is computed by replacing $E$ in Eq. 3 with the expression for $E_{D}$ in Eq. 6 . As a consequence, the computed evaporation rate reduces exactly to the diffusion-limited value under conditions for which there is no natural convection, and the number of fitting parameters in the correlation is reduced by one. The final form of the correlation is given by Eq. 9, which includes equivalent expressions for the correlation in terms of $R a$.

$$
\begin{aligned}
& S h=S h_{D}+c_{2}\left(\frac{\rho_{m}-\rho_{a}}{\rho_{a}}\right)^{n_{1}} S c^{n_{2}}\left(\frac{g R^{3}}{v_{a}^{2}}\right)^{n_{3}} \\
& S h=S h_{D}+\left(\frac{c_{2}}{\operatorname{Pr}^{n_{1}}}\right) R a^{n_{1}} S c^{n_{2}}\left(\frac{g R^{3}}{v_{a}^{2}}\right)^{\left(n_{3}-n_{1}\right)} \\
& S h=S h_{D}+\left(\frac{c_{2}}{\operatorname{Pr}^{n_{3}}}\right)\left(\frac{\rho_{m}-\rho_{a}}{\rho_{a}}\right)^{\left(n_{1}-n_{3}\right)} S c^{n_{2}} R a^{n_{3}}
\end{aligned}
$$

\section{Results and Discussion}

\subsection{Experimental Results}

The measured evaporation rates are presented in Fig. 3 as a function of the drop radius. Clearly, the evaporation rate is strongly dependent on the volatility and size of the drop. While the equilibrium vapor pressure provides a general indicator of volatility, it is not sufficient to predict the relative evaporation rates as two examples indicate. Methanol and heptane have approximately equal evaporation rates for most of the drop size range despite the fact that methanol has an equilibrium vapor pressure that is 2.7 times greater than that of heptane. Similarly, acetone's evaporation rates are nearly equal to those of $3 \mathrm{MP}$ even though acetone has a nearly $31 \%$ higher equilibrium vapor pressure. For the conditions of the experiments, for which the rate of evaporation is limited by the rate of vapor transport from the drop surface, the mass diffusivity and the density difference ratio, which drives natural convection, are important parameters. The density difference ratio of heptane is more than 8 times the value for methanol and presumably natural convection has a greater influence on the evaporation of heptane than it does on the evaporation of methanol. The same explanation may be applied to the comparison of the acetone and 3MP evaporation rates, though the contrast between the convective and diffusive contributions for these two species is less.

The influence of natural convection on the evaporation rate may be estimated with a simplifying assumption that the rates of vapor transport by diffusion and convection are independent and their sum equals the evaporation rate. The diffusion-limited evaporation rate, $E_{D}$, may be computed according to equations 6 and 7. Since there is not a simple solution for the vapor transport by natural convection, the convective contribution to the evaporation rate may be estimated by subtracting $E_{D}$ from the measured evaporation rate, $E_{m}$. The assumption that the diffusive transport is independent of the convective transport is a simplification since anything that affects the spatial distribution of the vapor concentration, as convection undoubtedly does, would affect the rate of diffusion. Despite this limitation, the difference $\left(E_{m}-E_{D}\right)$ is used as an indicator of convection's contribution to the evaporation rate and is plotted in Fig, 4. By comparing the data in Figs. 3 and 4, one can see that the values for $\left(E_{m}-E_{D}\right)$ suggest that convection accounts for $11 \%$ to $81 \%$ of the measured evaporation rate, depending on the species and drop size.

Figure 4 indicates that as the drop size increases, natural convection becomes a greater contributor to the evaporation rate. As drop radius increases from 1 to $22 \mathrm{~mm},\left(E_{m}-E_{D}\right)$ increases by a factor of 190 for 3MP and by a factor of 173 for heptane. For a given drop size, the order of $\left(E_{m}-E_{D}\right)$ roughly follows the order of $\left(\rho_{m}-\rho_{a}\right) / \rho_{a}$. Methanol has a very low density difference ratio and methanol's convective contribution to the evaporation rate as estimated by $\left(E_{m}-E_{D}\right)$ is very low, especially for drop radii equal to or less than $6 \mathrm{~mm}$. Exceptions to the order may be a result of the coupling of diffusive and convective transport. As reported in [16], a schlieren image which shows the vapor distribution above an evaporating hexane drop (radius $=6.5 \mathrm{~mm}$ ), indicates that the vapor is contained in a distinct "cloud" which is approximately flat, has a thickness of about $5 \mathrm{~mm}$, and spreads radially away from the drop. This distribution is much different than what would be expected for diffusion-limited evaporation of the same hexane drop, in which case the vapor would diffuse away from the drop in a roughly hemispherical direction and extend much further than $5 \mathrm{~mm}$ from the drop. Thus, by altering the distribution of the vapor, convection may have the effect of increasing the gradient in the vapor concentration, at least the vertical gradient above the drop, which would result in an increase in the diffusive transport of the vapor from the drop surface. This sort of coupling of diffusion and convection may explain why for radii greater than $6 \mathrm{~mm},\left(E_{m}-E_{D}\right)$ for methanol approaches the values for heptane whereas for smaller radii $\left(E_{m}-E_{D}\right)$ for methanol is much less than the values for heptane. It may be that at the larger radii, convection of the methanol vapor becomes significant enough to cause an increase in the vapor concentration gradient and thereby cause an increase in the rate of diff usion beyond the value predicted by equations 6 and 7. This scenario would result in an increase in $\left(E_{m}-E_{D}\right)$ which is not attributable solely to convection. 


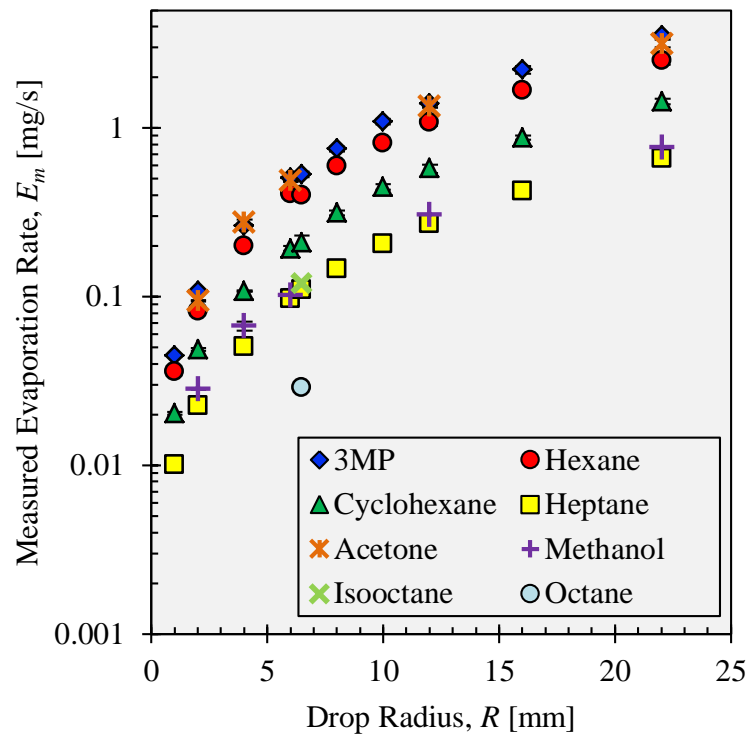

Figure 3. Plot of the measured evaporation rates as a function of drop size. The evaporation rates are plotted on a logarithmic scale to improve clarity. The measurement variation for each of the plotted values is smaller than the data symbol.

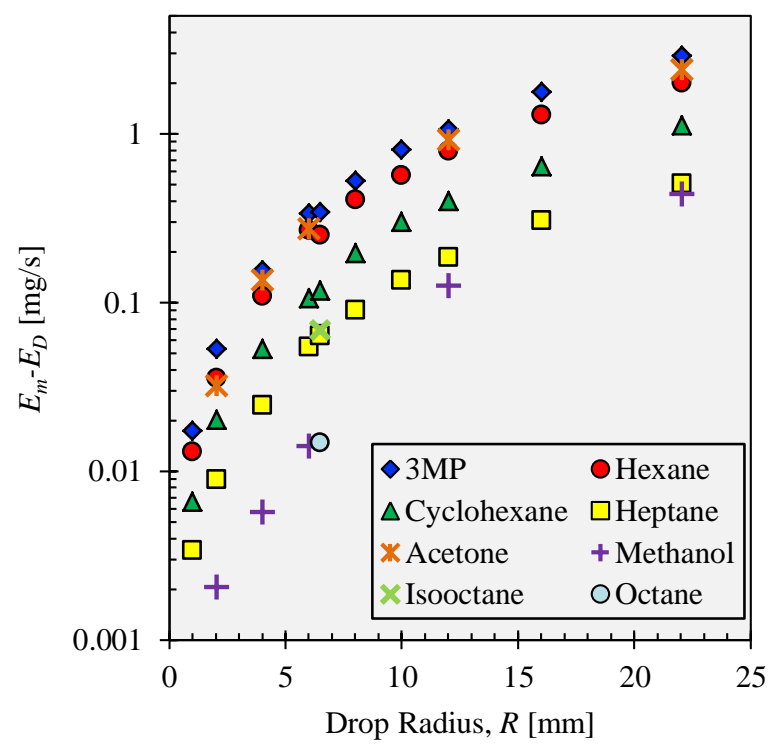

Figure 4. Plot of the difference between the measured and diffusion-limited evaporation rates as a function of drop size. The plotted differences are estimates of the convective contribution to the overall evaporation rate.

It is also plausible that diffusion could act to reduce the strength of convection by dispersing the vapor and keeping the density difference ratio low (much lower than the computed value). Since convection is a faster transport mechanism than is diffusion, one would expect the result of this sort of interaction to be a reduction in the evaporation rate and a commensurate reduction in $\left(E_{m}-E_{D}\right)$. This effect is an alternative explanation for methanol's trend for $\left(E_{m}-E_{D}\right)$ as a function of drop size. For drop radii less than $6 \mathrm{~mm}$, diffusion may be strong enough to keep the density difference ratio low, and thereby also the natural convection.

In actuality, both of the coupling effects between diffusion and convection (one tending to increase $E_{m}$ and the other to decrease it) may be significant under different conditions. For methanol drops of radius less than $6 \mathrm{~mm}$, diffusion may be strong enough to keep convection low, and for larger drops convection may be strong enough to affect the vapor distribution and increase the rate of diffusion by increasing the vapor concentration gradient. The transition from one effect to the other may explain the apparent jump in methanol's trend for $\left(E_{m}-E_{D}\right)$ versus drop size. Detailed computer modelling of the evaporation process and more experimental studies are needed to confirm or refute this explanation. Methanol's trend for $\left(E_{m}-E_{D}\right)$ versus drop size is the only 
one of the eight species studied that appears to contain a jump, which may suggest that none of the other species pass through a transition.

\subsection{Correlation Results}

Minitab statistical software was employed to compute the constants $c_{2}$ and $\mathrm{n}_{1}$ through $\mathrm{n}_{3}$ of Eq. 9 by nonlinear regression to fit $S h$ values computed from the measured evaporation rates by Eq. 3. The resulting correlation constants are presented in Table 3 along with the RMS error between the computed and measured evaporation rates. Two sets of constants are given, one set for a correlation fitted to the measurements of all eight species (Version A), and another set for a correlation that was fitted to a data set that included all but the methanol measurements (Version B).

\begin{tabular}{|c|c|c|c|c|c|}
\hline $\boldsymbol{c}_{2}$ & $\boldsymbol{n}_{\boldsymbol{1}}$ & $\boldsymbol{n}_{\mathbf{2}}$ & $\boldsymbol{n}_{\mathbf{3}}$ & $\begin{array}{c}\text { RMS Error } \\
(\boldsymbol{\%})\end{array}$ & $\begin{array}{c}\text { Correlation } \\
\text { Version }\end{array}$ \\
\hline 0.354 & 0.422 & 0.388 & 0.231 & 6.5 & $\mathrm{~A}$ \\
\hline 0.345 & 0.456 & 0.508 & 0.229 & 4.9 & $\mathrm{~B}$ \\
\hline
\end{tabular}

Table 3. Correlation constants for the nonlinear regression of Eq. 9 to $S h$ values computed from measured evaporation rates, and the resulting RMS error in the calculation of the evaporation rates compared to measured values. Methanol data was included in the fitted data for version A and excluded for version B.

Figure 5 shows the percentage error in the predicted evaporation rates for all of the conditions of the experiments for Version A of the correlation. Clearly, the correlation does a relatively poor job fitting the methanol data, for which the error approaches $20 \%$ at small radii and it approaches $-20 \%$ at large radii. However, with the exception of the methanol data, the correlation results in good agreement with the measured values. Excluding the methanol data, all but four of the computed values are within $\pm 8 \%$ of the measured values and nearly $60 \%$ of the computations have an error less than $\pm 5 \%$. The RMS error is $6.5 \%$. As a comparison, the RMS error of the correlation given by Eq. 1 is $12.7 \%$.

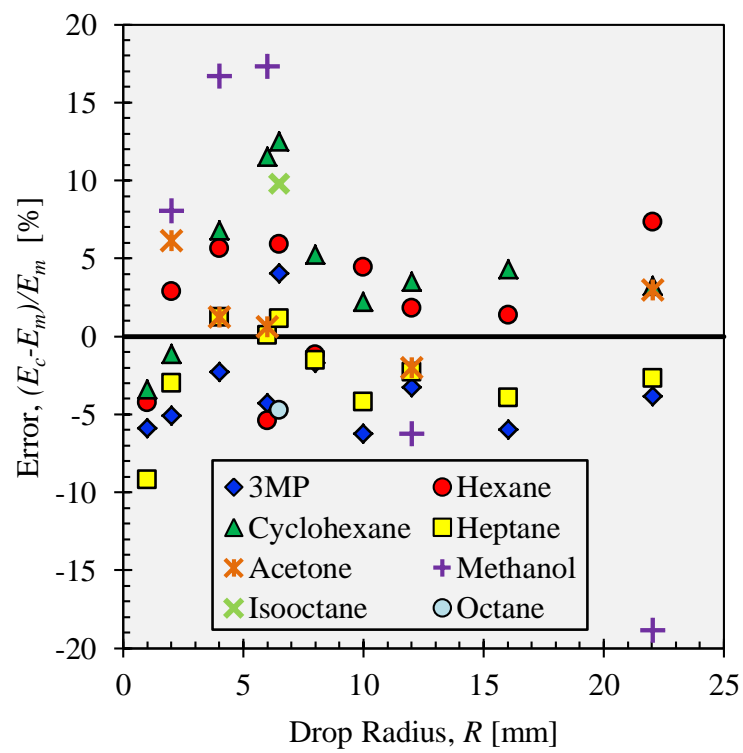

Figure 5. Percentage error in the computed evaporation rate for Version A of the correlation. The RMS error considering all of the data is $6.5 \%$.

The large error for methanol may be due to a difference in the nature of the dependence on drop size compared to the other species of the study. For constant temperature and pressure conditions, both the density difference ratio and $S c$ are constant for each species and Eq. 9 may be written as Eq. 10, where the density difference ratio and $S c$ are contained in the coefficient $a$. Equation 10 was fit to measured values for each species individually (except isooctane and octane for which measurements were obtained for just one drop size) and the values of the exponent $n_{3}$ are approximately equal $(0.238 \pm 0.010)$ for all of the species except for methanol, for which the value of $n_{3}(0.410)$ is 1.7 times greater.

$$
\left(S h-S h_{D}\right)=a\left(\frac{g R^{3}}{v_{a}^{2}}\right)^{n_{3}}
$$


Of the species in this study, methanol has the lowest $S c$ and the lowest density difference ratio. Methanol's $S c$ is from $38 \%$ to $100 \%$ lower than the values for the other species. After methanol's, the second lowest density difference ratio is estimated to be three times larger and the highest is more than 26 times larger. Perhaps the low $S c$ and/or low density difference ratio of methanol is below the range of applicability for a correlation that includes the other species of this study. (The $R a$ values for methanol fall within the range of $R a$ values for the other species.) It may be possible to extend the range of applicability of a correlation by making the exponent $n_{3}$ a function of one or more physical properties of the drop, though a suitable function involving the density difference ratio and $S c$ was not found for this study.

In an attempt to improve the correlation's fit for the species other than methanol, the nonlinear regression was conducted for a data set that excludes the methanol measurements, and the resulting correlation is Version B. The RMS error for Version B is $4.9 \%$. It is interesting to note that the error for Version B is equal to the RMS error for Version A if the methanol error is not counted. While there are small differences in the errors between the two versions for each of the species, the slight improvement in some species are offset by the slight worsening of others with no net change in the overall error. For comparison, the RMS error for Eq. 1 if methanol is excluded is $12.5 \%$. The most significant difference between Versions A and B is the exponent on $S c, n_{2}$. The other constants are nearly unchanged.

The results show that the correlation provides good agreement over the full range of conditions for all of the species except for methanol, for which the correlation agrees marginally with the measurements. Ra varies by nearly five orders of magnitude, from 3.4 to $1.3 \times 10^{5}$, which is much broader than any of the ranges of the $S h$ - $R a$ correlations reviewed by Bower and Saylor [8]. Sc varies from 1.0 to 2.2 if the methanol data is included or from 1.4 to 2.2 if methanol data is excluded, and the dimensionless size term $\left(\frac{g R^{3}}{v a^{2}}\right)$ varies by four orders of magnitude, from 38 to $3.9 \times 10^{5}$.

Equation (9) suggests that the influences of diffusion and natural convection are independent and additive with the diffusive effect computed by $S h_{D}$ and the convective effect computed by the second term of the expression, which is called the convective term. However, as previously discussed, the two transport mechanisms are coupled and consequently the convective term should be perceived as containing both the influence of natural convection and the net change in the influence of diffusion compared to the diffusion-only condition.

Converting Eq. 9 to dimensional form, the evaporation rate is given by Eq. 11. The first term on the right hand side is the diffusion-limited evaporation rate, which is defined by Eq. 6, and the second term is the dimensional form of the convective term. In this form of the correlation, the convective term's dependency on drop size, density difference ratio, and diffusivity may be investigated.

$$
E=E_{D}+c_{2} \pi g^{n_{3}} v_{a}^{\left(n_{2}-2 n_{3}\right)} R^{\left(3 n_{3}+1\right)}\left(\frac{P_{v} M}{R_{u} T}\right) D^{\left(1-n_{2}\right)}\left(\frac{\rho_{m}-\rho_{a}}{\rho_{a}}\right)^{n_{1}}
$$

The value for $n_{3}$ is 0.23 , which indicates that the effect of convection on the evaporation rate is dependent on the drop radius raised to the power 1.69. The value of the exponent for drop radius seems reasonable when one considers the value for diffusionlimited evaporation is 1 (see Eq. 6), and the value for forced convection is 2 , since in that case the evaporation rate is proportional to the area. Furthermore, the exponent value for radius is within the range of values computed for the $S h-R a$ correlations reviewed by Bower and Saylor [8], which are between 1.63 and 1.99 .

The density difference ratio, which is the driving factor for natural convection, is raised to the power of approximately 0.46 , which is higher than the exponent values on $R a$ for the correlations reviewed by Bower and Saylor, which are between 0.205 and 0.327 .

The convective term also includes a dependency on the mass diffusivity. For $n_{2}$ equal to 0.51 , the value for Version $\mathrm{B}$, the diffusivity is raised to the power 0.49 . This dependency is consistent with the hypothesis that natural convection and diffusion are coupled in such a way that convection increases the rate of diffusion.

Figure 6 is a plot of the measured and computed (Version B) evaporation rates as a function of drop size. Methanol is included for comparison. There is generally very good agreement between the computed and measured values, though for methanol the correlation over-predicts the evaporation rate for small drop radii and under predicts the rate for large radii. As shown in the figure, the correlation does a good job of matching the evaporation rates of isooctane and octane, which is encouraging since only one datum point for each of these two species was included in the set of 52 data points used to fit the correlation, and therefore the measurements for isooctane and octane had a very small influence on the nonlinear regression. 


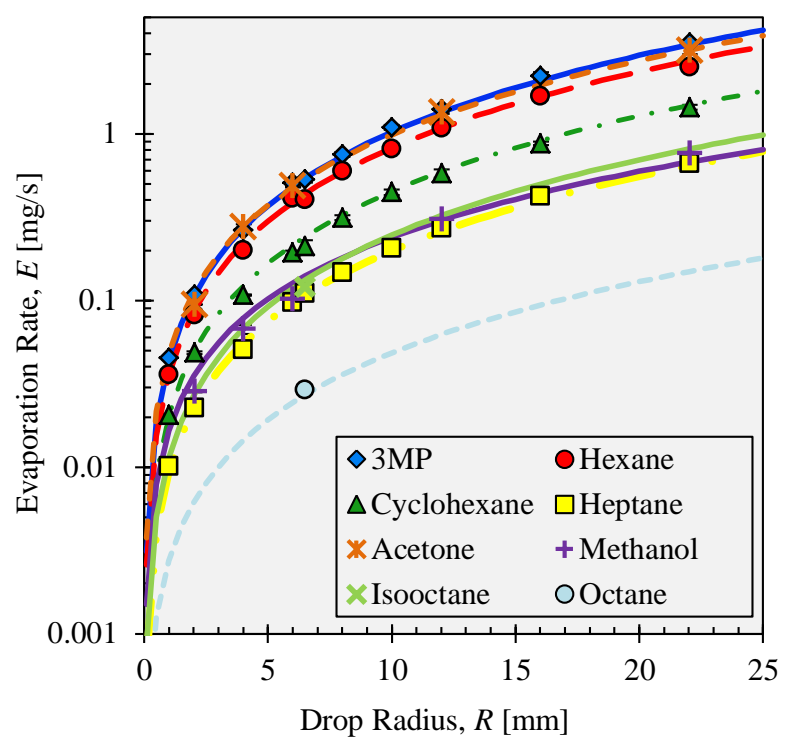

Figure 6. Measured and computed evaporation rates of each of the eight species studied as a function of drop size. Measured values are given by the datum symbols and the computed values by the lines. Error bars are included but are smaller than the datum symbols.

The lack of satisfactory agreement for methanol suggests that a limit of applicability for the new correlation may be a density difference ratio less than 0.052 (the value for octane) and/or $S c$ less than 1.4 (the value for acetone). More experiments are needed to better clarify this limit.

\section{Conclusions}

For all of the measured species except methanol, the evaporation rates have similar dependence on drop size. Consequently, while the new correlation fits well the measured evaporation rates of seven of the eight species studied, the correlation produces comparatively poor agreement with the measured values for methanol. Version A of the correlation, for which the methanol measurements were included in the set of fitted data, has a total RMS error of 6.5\%. If methanol is excluded from the error calculations, the RMS error reduces to $4.9 \%$. A negligible improvement in the correlation is obtained when methanol is excluded from the set of fitted data (Version B), though the convective term's dependence on diffusivity is reduced. We are not certain why the dependence on diffusivity is stronger for Version A, but it may be a compensation for methanol's very low density difference ratio.

By intent, the correlation is meant to provide a simple expression for the evaporation rate under combined diffusion and convection conditions. Though simple in form, the correlation provides insight into the nature of the complex coupling between the effects of the two transport mechanisms. The correlation is composed of two terms, the evaporation rate under diffusionlimited conditions and the evaporation rate due to the effects of natural convection, which includes the net change in transport by diffusion. The coupling of the two transport mechanisms is indicated by the dependency of the second, convective term on mass diffusivity. It is speculated that the nature of the coupling is competitive in such a way that when diffusion is relatively strong, it acts to suppress convection but when convection is relatively strong, it results in an increase in diffusion. The simple form of the correlation is not able to capture this competitive nature of the coupling. Instead, the correlation appears to capture just the increase in diffusive transport as a consequence of convection, which is indicated by the dependency of the convective term on mass diffusivity raised to the power of 0.49 . There is no means in the correlation for the effect of convection to be reduced by diffusion. This limitation may be another reason why the correlation does a comparatively poor job of fitting the methanol data.

Over the broad range studied, the convection term increases with drop size at a rate approximately equal to the rates given by previous $S h-R a$ correlations, but the term has a stronger dependence on the density difference ratio than the other correlations have. By fitting the measurements to an equation containing separate factors for the density difference ratio and drop size, rather than forcing those factors to be contained solely in $R a$, better agreement is obtained and for a broader range of conditions than what is obtained by previous correlations. Inclusion of a separate term for diffusion-limited evaporation also helped to improve the accuracy of the correlation. 


\section{Acknowledgements}

Acknowledgement is made to the Donors of the American Chemical Society Petroleum Research Fund for support of this research. We are also grateful to Dr. Cabral Balreira for an informative discussion regarding non-linear regression, and to Adhwaith Mani and Travis Benavides for their assistance collecting data and investigating correlations.

\section{References}

[1] Dunn, G.J., Wilson, S.K., Duffy, B.R., David, S., and Sefiane, K., A Mathematical Model for the Evaporation of a Thin Sessile Liquid Droplet: comparison between Experiment and Theory, Colloid Surface A, 323, 2008, 50-55.

[2] Semenov, S., Starov, V.M., Rubio, R.G., and Velarde, M.G., Instantaneous Distribution of Fluxes in the course of Evaporation of Sessile Liquid Droplets: Computer Simulations, Colloid Surface A, 372, 2010, 127-134.

[3] $\mathrm{Hu}, \mathrm{H}$. and Larson, R.G., Evaporation of a Sessile Droplet on a Substrate, J Phys Chem B, 106, 2002, 1334-1444.

[4] Poulard, C., Guéna, G., Cazabat, A.M., Boudaoud, A., Amar, M.B., Rescaling the Dynamics of Evaporating Drops, Langmuir 21, 2005, 8226-8233.

[5] Thomas, N., Ferguson, A., Evaporation from a Circular Water Surface, Philos Mag, 34, 1917, 309-321.

[6] Gauss, A., Fuel and Hydrocarbon Vaporization, BRL Report 1661, Aberdeen Proving Ground, Aberdeen, MD, 1973.

[7] Poulard, C., Guéna, G., Cazabat, A.M., Diffusion-Driven Evaporation of Sessile Drops, J Phys-Condens Mater, 17, 2005, S4213-S4227.

[8] Bower, S.M., Saylor, J.R., A Study of the Sherwood-Rayleigh Relation for Water undergoing Natural Convection-Driven Evaporation, Int J Heat Mass Transfer, 52, 2009, 3055-3063.

[9] Bower, S.M., Saylor, J.R., Erratum to "A Study of the Sherwood-Rayleigh Relation for Water undergoing Natural Convection-Driven Evaporation”, Int J Heat Mass Transfer, 54, 2011, 749.

[10] Sparrow, E.M., Kratz, G.K., Schuerger, M.J., Evaporation of Water from a Horizontal Surface by Natural Convection, $J$ Heat Trans-T ASME, 105, 1983, 469-475.

[11] Boelter, L.M.K., Gordon, H.S., Griffin, J.R., Free Evaporation into Air of Water from a Free Horizontal Quiet Surface, Ind Eng Chem, 38 (6), 1946, 596-600.

[12] Sharpley, B.F., Boelter, L.M.K., Evaporation of Water into Quiet Air from a One-Foot Diameter Surface, Ind Eng Chem, 30 (10), 1938, 1125-1131.

[13] Jodat, A., Moghiman, M., Anbarsooz, M., Experimental Comparison of the ability of Dalton Based and Similarity Theory Correlations to Predict Water Evaporation Rate in Different Convection Regimes, Heat Mass Transfer, 48 (8), 2012,1397 1406.

[14] Jodat, A., Moghiman, M., An experimental Assessment of the Evaporation Correlations for Natural, Forced and Combined Convection Regimes, P I Mech Eng C-J Mec, 226 (1), 2012, 145-153.

[15] Shah, M.M., Rate of Evaporation from Undistrubed Water Pools to Quiet Air: Evaluation of Available Correlations, Int J $H V A C \& R, \mathbf{8}, 2002,125-131$.

[16] Kelly-Zion, P.L., Pursell, C.J., Booth, R.S., VanTilburg, A.N., Evaporation Rates of Pure Hydrocarbon Liquids under the influences of Natural Convection and Diffusion, Int J Heat Mass Transfer, 52, 2009, 3305-3313.

[17] Kelly-Zion, P.L., Pursell, C.J., Vaidya, S., and Batra, J., Evaporation of Sessile Drops under Combined Diffusion and Natural Convection, Colloid Surface A, 381, 2011, 31-36.

[18] C.L. Yaws, Thermodynamic and Physical Property Data, Gulf Publishing Company, Houston, TX, 1992.

[19] Poling, B.E., Prausnitz, J.M., O’Connell, J.P., The Properties of Gases and Liquids, $5^{\text {th }}$ Ed., New York, McGraw-Hill, 2001. 


\section{Table Captions:}

Table 1. Thermophysical properties of the hydrocarbon species at $23.3^{\circ} \mathrm{C}$ and $96 \mathrm{kPa}$.

Table 2. Percent error resulting from the use of Eqs. 1 and 2 to compute the evaporation rates of isooctane and octane for a drop radius of $6.5 \mathrm{~mm}$.

Table 3. Correlation constants for the nonlinear regression of Eq. 9 to $S h$ values computed from measured evaporation rates, and the resulting RMS error in the calculation of the evaporation rates compared to measured values. Methanol data was included in the fitted data for version A and excluded for version B.

\section{Figure Captions:}

Figure 1. Schematic of a profile view of a sessile drop on the disk-shaped substrate. The drop is attached around the circular periphery of a short platform located at the center of the substrate, and in this way the drop size is maintained during evaporation.

Figure 2. Comparison of the percentage error in the computed evaporation rates from previously published correlations given by Eq. 1 (Fig. 2a) and Eq. 2 (Fig. 2b). The RMS errors are 12.7\% for Eq. 1 and $70.1 \%$ for Eq. 2.

Figure 3. Plot of the measured evaporation rates as a function of drop size. The evaporation rates are plotted on a logarithmic scale to improve clarity. The measurement variation for each of the plotted values is smaller than the data symbol.

Figure 4. Plot of the difference between the measured and diffusion-limited evaporation rates as a function of drop size. The plotted differences are estimates of the convective contribution to the overall evaporation rate.

Figure 5. Percentage error in the computed evaporation rate for Version A of the correlation. The RMS error considering all of the data is $6.5 \%$.

Figure 6. Measured and computed evaporation rates of each of the eight species studied as a function of drop size. Measured values are given by the datum symbols and the computed values by the lines. Error bars are included but are smaller than the datum symbols. 